\title{
Violência Comunitária e Transtorno de Estresse Pós-Traumático em Crianças e Adolescentes
}

\author{
Community Violence and Posttraumatic Stress Disorder \\ in Children and Adolescents
}

\author{
Liana Furtado Ximenes, ${ }^{*}$, , Simone Gonçalves de Assis ${ }^{\mathrm{b}}$, Thiago de Oliveira Pires ${ }^{\mathrm{b}}$ \\ \& Joviana Quintes Avanci ${ }^{\mathrm{b}}$ \\ a Ministério da Saúde e Universidade Estácio de Sá, Rio de Janeiro, Rio de Janeiro, Brasil \\ $\&^{b}$ Fundação Oswaldo Cruz, Rio de Janeiro, Rio de Janeiro, Brasil
}

\begin{abstract}
Resumo
O presente estudo tem como objetivo analisar a associação entre exposição à violência comunitária e o Transtorno de Estresse Pós-Traumático (TEPT) segundo gênero e faixa etária entre crianças e adolescentes de escolas públicas de São Gonçalo/RJ. Participaram da análise deste artigo 399 crianças e adolescentes (idade média de 10,6 anos, $D P=1,0$ ). A prevalência total de sintomas de TEPT nas crianças e adolescentes é de $9,5 \%$, sendo maior nas meninas $(12,6 \%)$ do que nos meninos $(6,5 \%)$. No modelo multivariado, destaca-se que as crianças menores de 10 anos têm duas vezes mais chances de desenvolverem sintomas deste transtorno que as mais velhas e que quanto maior a exposição à violência comunitária maiores as chances de surgimento de sintomas de TEPT.

Palavras-chave: Transtorno de Estresse Pós-Traumático, violência comunitária, crianças, adolescentes.
\end{abstract}

\begin{abstract}
This study aims to analyze the association between exposure to community violence and Posttraumatic Stress Disorder (PTSD) by gender and age among children and adolescents of public schools in São Gonçalo-RJ. Three hundred and ninety-nine children and adolescents participated in this analysis (mean age 10.6 years; $S D=1.0$ ). The prevalence of PTSD symptoms in children and adolescents is $9.5 \%$, being higher among girls $(12.6 \%)$ than among boys $(6.5 \%)$. In the multivariate model, it is emphasized that children under 10 years old are twice as likely to develop symptoms of this disorder, than older ones; and the higher exposure to community violence, the emergence of PTSD symptoms is more likely.

Keywords: Posttraumatic stress disorder, community violence, children, adolescents.
\end{abstract}

Crianças e adolescentes de grandes centros urbanos estão frequentemente expostos à violência comunitária. Revisões de literatura internacionais citam prevalências variadas desta vitimização, podendo oscilar entre 3-96\% em crianças e adolescentes de vários países do mundo (Fowler, Tompsett, Braciszewski, Jacques-Tiura, \& Baltes, 2009; Margolin \& Gordis, 2000). A ampla variação pode ser explicada pela utilização de diferentes instrumentos de aferição, pelas fontes variadas para avaliar a vitimização (relato da criança/adolescente, dos pais ou professores e estatísticas criminais, por exemplo), pela abordagem de diferentes tipos de vitimização (direta e indireta) e pela real maior exposição da violência comunitária, decorrente de estudos realizados em áreas de conflito intenso.

A violência comunitária não tem sido definida clara ou consistentemente, o que também dificulta comparações de

* Endereço para correspondência: Fundação Oswaldo Cruz, Av. Brasil, 4036, sala 700, Manguinhos, Rio de Janeiro, RJ, Brasil 21040-361. E-mail: liana@claves. fiocruz.br resultados entre estudos. De forma geral, o seu foco está na violência vivenciada ou testemunhada pela criança/ adolescente no ambiente da rua, da escola e de outros locais proximais em que transita (Sieger, Rojas-Vilches, Mckinney, \& Renk, 2004). Inclui situações como ser vítima/testemunha de ações de tráfico de drogas, uso de armas, ver pessoas mortas, tiroteios, assassinatos e roubos.

Pesquisas no Brasil apontam a gravidade deste problema. Um estudo realizado em município do estado do Rio de Janeiro estima que $27,3 \%$ das crianças e adolescentes são expostos à violência comunitária (Assis, Avanci, Pesce, Oliveira, \& Ximenes, 2007). Um outro realizado em dez capitais brasileiras demonstrou que $12 \%$ dos jovens sofreu agressão física na comunidade e que $16 \%$ foi ou teve um parente ameaçado de morte (Cardia, 1999).

A exposição à violência comunitária está associada a inúmeros prejuízos ao desenvolvimento e a saúde mental de crianças e adolescentes, tais como: problemas de comportamento externalizantes (comportamento agressivo e violação de regras); depressão e outros problemas de comportamento internalizantes (Assis, Avanci, \& Oliveira, 
2009; Margolin \& Gordis, 2000); além do Transtorno de Estresse Pós-Traumático (TEPT), que, pela condição de violência ser um dos determinantes de sua ocorrência, tem sido destacado dentre os problemas (Fowler et al., 2009; Shields, Nadasen, \& Pierce, 2008).

O Transtorno de Estresse Pós-Traumático caracteriza-se pelo aparecimento de sintomas de ansiedade após a exposição a um evento traumático, que foi vivenciado com medo e horror. Tal exposição envolve a experiência direta a um evento traumático ou o testemunho ou o conhecimento de um evento ameaçador à integridade pessoal. Os sintomas de TEPT incluem a revivência persistente de tal evento; a esquiva de estímulos associados ao trauma e de entorpercimento da reatividade geral; e o aumento da excitabilidade (Associação de Psiquiatria Americana [APA], 2002).

Gabbay, Oatis, Silva e Hirsch (2004) mostram prevalência de sintomas de TEPT ao longo da vida para crianças e adolescentes entre $2 \%$ a $9 \%$ e discutem que taxas mais elevadas são encontradas naqueles que vivenciaram experiências traumáticas específicas, como por exemplo, violência, desastres e acidentes. Em uma revisão realizada por Salzinger, Feldman, Stockhammer e Hood (2002) a prevalência entre adolescentes escolares norte-americanos oscila entre $29 \%$ a $34,5 \%$, sendo constatada associação com a violência comunitária.

No Brasil, dados sobre este tema na população infanto-juvenil são escassos e a maior parte se debruça na investigação da população adulta. Entre crianças e adolescentes, Ximenes, Oliveira e Assis (2009) verificaram que 10,8\% das crianças escolares investigadas apresentavam sintomas de TEPT, sendo que 6,5\% denotavam sintomas clínicos. Outros trabalhos brasileiros sobre o tema discutem sobre os aspectos teóricos do transtorno e de seu tratamento em crianças (Borges \& Dell'Aglio, 2008; Passarela, Mendes, $\&$ Mari, 2010).

Especialistas discutem a respeito da gravidade e especificidades do TEPT em faixas etárias mais novas, uma vez que na infância e adolescência seus sintomas costumam não desaparecer sem tratamento (Scheeringa, 2008). Crianças e adolescentes podem se sentir confusos, tristes ou "congelados" durante a exposição do evento traumático e apresentarem culpa e vergonha. Assim, Scheeringa, Zeanah e Cohen (2010) sugerem mudanças mais sensíveis nos critérios de TEPT na infância e adolescência, o que poderia alterar prevalências de $1,7 \%$ para $10,0 \%$ para crianças de 2 a 10 anos.

Outrossim, a vulnerabilidade de TEPT é maior no sexo feminino (March, Amaya-Jackson, Terry, \& Constanzo, 1997; Tolin \& Foa, 2006). Depois da exposição a um evento traumático, elas têm risco cinco vezes maior de desenvolverem sintomas do transtorno que o sexo masculino (Abanilla, 2004; Foster, Kuperminc, \& Price, 2004).

Além do maior conhecimento de um tema pouco conhecido em território nacional, a relevância deste estudo recai na fonte de informação - a própria criança/adolescente - uma vez que os estudos internacionais sobre violência comunitária e TEPT em crianças e adolescentes costumam partir de informações de pais ou outros cuidadores, fornecendo dados de um ponto de vista específico, podendo de alguma forma subestimar a situação do transtorno (Richters \& Martinez, 1993). A investigação do fenômeno a partir da informação de crianças e adolescentes propicia dados mais precisos sobre a vivência dos eventos violentos ocorridos na localidade em que crescem e seu possível impacto na saúde mental.

Partindo deste contexto, o presente estudo tem como objetivo analisar a associação entre a exposição da violência comunitária e sintomas de TEPT segundo gênero e faixa etária de crianças e adolescentes de escolas públicas municipais de São Gonçalo - cidade integrante da região metropolitana do estado do Rio de Janeiro.

\section{Método}

Este artigo apresenta dados seccionais da terceira etapa de um estudo longitudinal desenvolvido com 500 crianças selecionadas em 2005 através de um desenho amostral do tipo conglomerado nos seguintes estágios de seleção: no primeiro estágio, 25 escolas foram amostradas através da seleção do tipo proporcional ao tamanho, considerando o número de alunos de cada escola como variável auxiliar a seleção; na segunda etapa, utilizou-se amostra aleatória simples de duas turmas de $1^{\mathrm{a}}$ série do ensino fundamental dentro de cada escola sorteada; finalmente, sorteou-se aleatoriamente 10 alunos para cada uma das duas turmas amostradas nas 25 escolas. Os outros dois momentos da pesquisa aconteceram em 2006 e 2008 e os dados analisados no presente artigo correspondem a este último ano, com informações provenientes dos responsáveis e das crianças/adolescentes.

Em 2008, 66 crianças/adolescentes não participaram do estudo e outras 20 foram excluídas da análise por terem nível de inteligência intelectualmente deficiente (WISC; Wechsler, 2002), dificultando a avaliação de problemas emocionais e comportamentais como TEPT. Outros dois participantes não foram aqui analisados devido ao preenchimento incompleto das escalas que aferem TEPT e violência comunitária, bem como 13 crianças/adolescentes que apresentaram pontos extremos (outliers) na escala de violência comunitária. São, portanto, 399 crianças/ adolescentes aqui analisados.

A pesquisa foi aprovada pelo Comitê de Ética da Escola Nacional de Saúde Pública/Fundação Oswaldo Cruz, com o número de 57/09, CAAE: 0057.0.031.000-09. Foram encaminhados para tratamento em saúde mental as crianças e os adolescentes que apresentaram sintomas em nível clínico do Transtorno de Estresse Pós-Traumático, e para atendimento em serviço especializado em violência aquelas com histórico desta vitimização.

\section{Instrumentos}

Foram obtidas informações sobre o sexo e idade $(\leq 10$ ou $>$ de 10 anos) dos participantes, que serviram de base 
Ximenes, L. F., Assis, S. G., Pires, T. O. \& Avanci, J. Q. (2013). Violência Comunitária e Transtorno de Estresse Pós-Traumático em Crianças e Adolescentes.

para a categorização dos dados realizada nesse artigo. Também foram indagadas a escolaridade do responsável feminino da criança/adolescente, a estrutura familiar (quem vive com a criança/adolescente) e a renda per capita. Estes dados foram obtidos através de entrevista com pais/responsáveis das crianças/adolescentes amostrados.

As informações a respeito dos sintomas de TEPT e da violência na comunidade foram obtidas através da realização de perguntas às crianças/adolescentes, que incluíram as escalas descritas a seguir. A estratégia de aplicação dos questionários através da leitura pelos pesquisadores foi tomada para facilitar a compreensão das questões por crianças mais novas e das que apresentam maiores limitações para leitura e compreensão das questões.

A percepção da criança/adolescente sobre exposição da violência em casa, na escola, na comunidade e em outros lugares por ela frequentados foi aferida através da escala "Coisas que vi e ouvi" (CVO [Things I have seen and heard]; Assis, 2009; Richters \& Martinez, 1990). Compõe-se de 20 itens com cinco opções de respostas que variam do nunca a muitas vezes (pontuação de 0 a 4). Neste artigo apenas 12 itens referentes à violência comunitária são utilizados (Figura 1), assim como realizado por Malik (2008). Para obtenção do escore foram somadas as frequências dos itens, multiplicadas pela razão entre 12 (total de itens) e o total de itens válidos respondidos. $\mathrm{O}$ escore se refere tanto a variedade de eventos que a criança/adolescente vivencia como a frequência dos mesmos. Verificou-se confiabilidade adequada desta escala com $\alpha$ Cronbach de 0,763 .

A avaliação dos sintomas de TEPT foi realizada através da escala Youth Self-Report - YSR (Achenbach \& Rescorla, 2001; Bordin, Mari, \& Caeiro, 1995), que é um instrumento voltado para avaliação de problemas de comportamentos a ser preenchido pela própria criança ou adolescente. Foram utilizados 20 itens da escala para aferir sintomas de TEPT (Ruggiero \& McLeer, 2000; Wolfe, Gentile, \& Wolfe, 1989), como por exemplo: náuseas, enjôos por nervoso; dores de estômago/barriga por nervoso; problemas com o sono; mudança repentina de humor, mal humor, irritar-se com facilidade e; nervoso/ tenso. As opções de resposta da escala são: falso, pouco verdadeiro, muito verdadeiro; com escores que variam de 0 a 2 . A soma dos escores é padronizada através do escore $T$ para definição dos casos clínicos, limítrofes e não clínico (escore maior que 65 corresponde aos casos limítrofes ou clínicos). Estudo nacional informa adequada confiabilidade da CBCL/TEPT (versão da YSR respondida pelos pais/ responsáveis) com $\alpha$ Cronbach de 0,77 , porém encontrou sensibilidade baixa (40\%) entre a escala CBCL/TEPT e K-SADS-PL (Brasil, 2003) e especificidade de 73\% (Ximenes et al., 2009).

\section{Análise dos Dados}

Buscou-se verificar a associação do escore da escala CVO no âmbito da comunidade, controlada pelas variáveis de sexo e idade. A análise de associação entre TEPT e cada item da escala CVO foi igualmente estratificada por sexo. O nível de significância considerado foi de $5 \%$, utilizando o teste $F$ ajustado. O pacote estatístico utilizado foi o SPSS versão 15 .

Também foram ajustados modelos de regressão logística univariados e múltiplo, e avaliados os termos de interação (nível de significância de $5 \%$ ). O pacote estatístico utilizado foi o R versão 2.11.1.

As informações referentes ao desenho amostral foram consideradas em toda a análise (ajustes de modelo e testes de associação) para correção da precisão e incorporação de pesos amostrais visando à correção das medidas pontuais (Pessoa, Nascimento Silva, \& Duarte, 1997).

\section{Resultados}

A idade das 399 crianças e adolescentes estudados varia de 9 a 16 anos $(M=10,6$ anos e $D P=1,0)$. Do total, $50,4 \%$ são do sexo masculino; $25,3 \%$ têm a cor da pele branca e $69,5 \%$ preta ou parda. Quanto à escolaridade dos pais/responsáveis, $44,2 \%$ das mães e $46,3 \%$ dos pais têm ensino fundamental completo ou acima e; $13 \%$ das mães e $6 \%$ dos pais estão desempregados. Somente $7,2 \%$ das famílias tem renda per capita por mês maior que um salário mínimo em 2008 (R\$ 415,00), enquanto 61,9\% reportaram renda de menos de $1 / 2$ salário mínimo mensal por pessoa. Em termos de configuração familiar, 50,9\% das crianças/ adolescentes vivem com ambos os pais, $28 \%$ com somente um deles, $15,5 \%$ com um dos pais e madrasta/padrasto e $5,6 \%$ com outros parentes.

\section{Exposição à Violência Comunitária}

Na Figura 1 observam-se os tipos de violências investigados e a frequência de sua vitimização relatada pelas crianças e adolescentes. Constata-se a alta exposição à violência comunitária entre os participantes: $86,2 \%$ vivenciaram pelo menos uma de suas formas. Destas, $21,3 \%$ sofreram apenas um tipo de violência, $34,3 \%$ sofreram de 2 a 3 tipos e 30,6\% de 4 a 9 tipos. Os mais citados, em especial, foram: a convivência com tiros ouvidos na comunidade, a proximidade com bandidos, a observação do corpo de pessoas mortas e o testemunho de pessoas serem presas e espancadas no local em que moram.

Quando se avalia a exposição à violência de acordo com a faixa etária, percebe-se que os mais velhos presenciaram mais pessoas serem presas $(32,0 \%$ daqueles com dez ou mais anos e $22,8 \%$ das crianças mais novas; $p=0,033)$, tráfico de drogas $(29,7 \%$ e $13,5 \%$ respectivamente; $p=0,003)$ e viram mais pessoas mortas na comunidade $(44,3 \%$ e $26,8 \%$, respectivamente; $p=0,005$; Tabela 1).

Quanto à exposição à violência comunitária segundo o sexo do entrevistado, apenas uma questão mostra-se distinta: ver alguém ser preso, mais comum entre meninos $(31,4 \%$ versus $22,2 \%$ entre meninas; $p=0,023$; Tabela 1). 


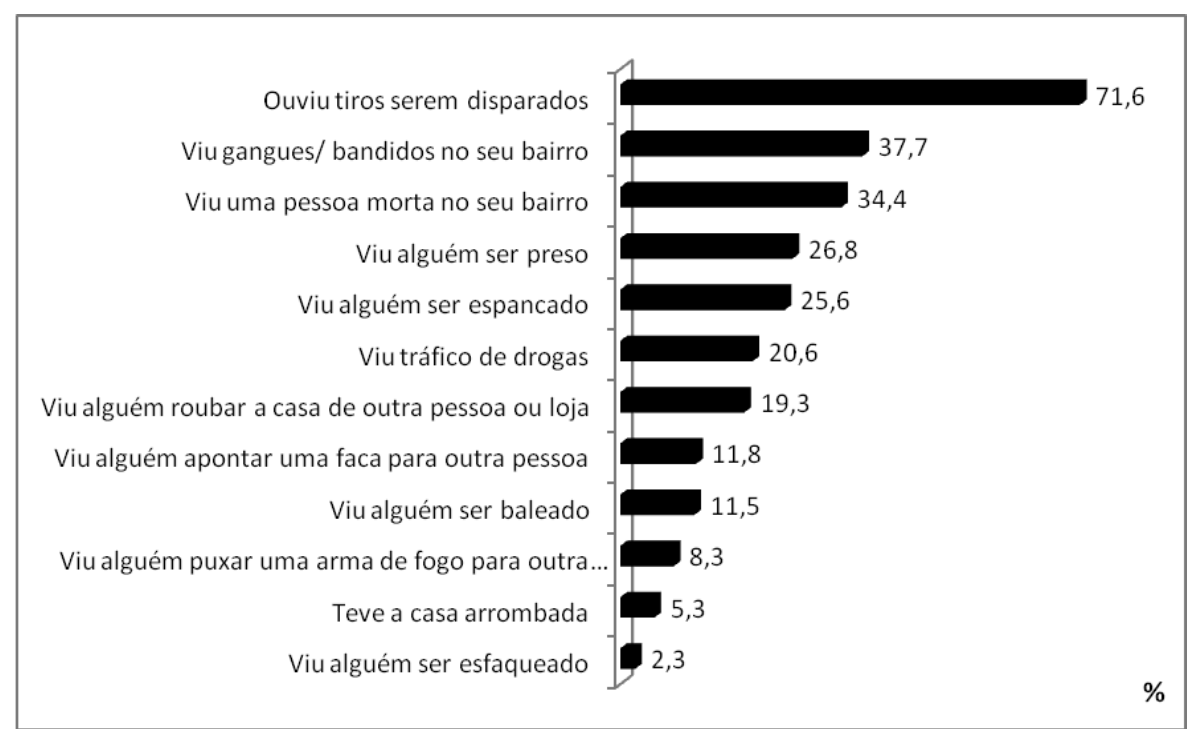

Figura 1. Prevalência da exposição à violência comunitária em crianças e adolescentes de escolas municipais de São Gonçalo - RJ $(N=399)$.

Tabela 1

Prevalência da Exposição à Violência Comunitária em Crianças e Adolescentes Escolares, segundo Sexo e Faixa Etária, São Gonçalo - RJ (N=399)

\begin{tabular}{|c|c|c|c|c|c|c|c|}
\hline & & \multicolumn{2}{|c|}{ Sexo } & \multirow{2}{*}{$P$-valor } & \multicolumn{2}{|c|}{ Idade } & \multirow{2}{*}{$P$-valor } \\
\hline & & Mas & Fem & & $<=10$ & $>10$ & \\
\hline \multirow[t]{2}{*}{ Você ouviu tiros serem disparados? } & $n$ & 146 & 139 & 0,664 & 156 & 129 & 0,438 \\
\hline & $\%$ & 72,6 & 70,6 & & 70,0 & 73,7 & \\
\hline \multirow[t]{2}{*}{ Você viu alguém ser preso? } & $n$ & 63 & 44 & $0,023^{*}$ & 51 & 56 & $0,033^{*}$ \\
\hline & $\%$ & 31,4 & 22,2 & & 22,8 & 32,0 & \\
\hline \multirow[t]{2}{*}{ Você viu tráfico de drogas? } & $n$ & 41 & 41 & 0,961 & 30 & 52 & $0,003 * *$ \\
\hline & $\%$ & 20,5 & 20,7 & & 13,5 & 29,7 & \\
\hline \multirow[t]{2}{*}{ Você viu alguém ser espancado? } & $n$ & 52 & 50 & 0,907 & 55 & 47 & 0,612 \\
\hline & $\%$ & 25,9 & 25,3 & & 24,6 & 26,9 & \\
\hline \multirow[t]{2}{*}{ Você viu alguém ser esfaqueado? } & $n$ & 6 & 3 & 0,337 & 4 & 5 & 0,517 \\
\hline & $\%$ & 3,0 & 1,5 & & 1,8 & 2,9 & \\
\hline \multirow[t]{2}{*}{ Você viu alguém ser baleado? } & $n$ & 30 & 16 & 0,115 & 22 & 24 & 0,252 \\
\hline & $\%$ & 14,9 & 8,1 & & 9,8 & 13,7 & \\
\hline \multirow[t]{2}{*}{ Você viu uma pessoa morta no seu bairro? } & $n$ & 72 & 65 & 0,546 & 60 & 77 & $0,005^{* *}$ \\
\hline & $\%$ & 35,8 & 32,9 & & 26,8 & 44,2 & \\
\hline \multirow[t]{2}{*}{ Você viu gangues/ bandidos no seu bairro? } & $n$ & 69 & 81 & 0,198 & 75 & 75 & 0,108 \\
\hline & $\%$ & 34,5 & 40,9 & & 33,7 & 42,8 & \\
\hline \multirow[t]{2}{*}{ Você viu alguém puxar uma arma de fogo para outra pessoa? } & $n$ & 22 & 11 & 0,143 & 17 & 16 & 0,590 \\
\hline & $\%$ & 10,9 & 5,6 & & 7,6 & 9,2 & \\
\hline \multirow[t]{2}{*}{ Sua casa foi arrombada? } & $n$ & 11 & 10 & 0,872 & 10 & 11 & 0,407 \\
\hline & $\%$ & 5,5 & 5,1 & & 4,4 & 6,3 & \\
\hline \multirow[t]{2}{*}{ Você viu alguém apontar uma faca para outra pessoa? } & $n$ & 18 & 29 & 0,080 & 27 & 20 & 0,844 \\
\hline & $\%$ & 9,0 & 14,6 & & 12,1 & 11,4 & \\
\hline \multirow[t]{2}{*}{$\begin{array}{l}\text { Você viu alguém roubar alguma coisa da casa de outra pessoa } \\
\text { ou de uma loja? }\end{array}$} & $n$ & 36 & 41 & 0,465 & 40 & 37 & 0,389 \\
\hline & $\%$ & 17,9 & 20,7 & & 17,9 & 21,1 & \\
\hline
\end{tabular}


Ximenes, L. F., Assis, S. G., Pires, T. O. \& Avanci, J. Q. (2013). Violência Comunitária e Transtorno de Estresse Pós-Traumático em Crianças e Adolescentes.

$\mathrm{Na}$ análise da $\mathrm{CVO}$, constata-se que o escore oscilou entre 0 e 22 pontos e que a média encontrada foi de 5,9 $(D P=5,4)$ itens de violência comunitária. Isso indica que as crianças e os adolescentes convivem com uma variedade de tipos de violência na comunidade em que vivem e/ou são vítimas de uma frequência significativa de alguns desses eventos no seu dia a dia.

\section{Prevalência de TEPT e a Associação com a Violência Comunitária}

A prevalência total de sintomas de TEPT nas crianças e adolescentes foi de 9,5\%, sendo maior nas meninas $(12,6 \%)$ do que nos meninos (6,5\%). Cerca de $11 \%$ das crianças mais novas e $7,4 \%$ das mais velhas apresentaram sintomas do transtorno.

Tabela 2

Estimativa dos Coeficientes, Erro-Padrão, Razão de Chances e Significância dos Modelos

\begin{tabular}{|c|c|c|c|c|c|c|c|c|c|}
\hline \multirow[b]{2}{*}{ Variável } & \multicolumn{3}{|c|}{ Modelos simples } & \multicolumn{3}{|c|}{ Modelo múltiplo } & \multicolumn{3}{|c|}{ Modelo múltiplo com interação } \\
\hline & $\begin{array}{c}\beta^{1} \\
\left(\mathrm{EP}^{2}\right)\end{array}$ & $\begin{array}{c}\mathrm{OR}^{3} \\
\exp (\beta)\end{array}$ & $P$-valor & $\begin{array}{c}\beta^{1} \\
\left(\mathrm{EP}^{2}\right)\end{array}$ & $\begin{array}{c}\mathrm{OR}^{3} \\
\exp (\beta)\end{array}$ & $P$-valor & $\begin{array}{c}\beta^{1} \\
\left(\mathrm{EP}^{2}\right)\end{array}$ & $\begin{array}{c}\mathrm{OR}^{3} \\
\exp (\beta)\end{array}$ & $P$-valor \\
\hline Sexo feminino & $\begin{array}{c}0,737 \\
(0,391)\end{array}$ & 2,090 & 0,073 & $0,743(0,396)$ & 2,102 & 0,075 & $-0,285(0,434)$ & 0,752 & 0,653 \\
\hline Idade $<=10$ & $\begin{array}{c}0,444 \\
(0,361)\end{array}$ & 1,559 & 0,231 & $0,697(0,329)$ & 2,009 & 0,046 & $0,719(0,343)$ & 2,053 & 0,049 \\
\hline $\begin{array}{l}\text { Violência } \\
\text { comunitária }\end{array}$ & $\begin{array}{c}0,093 \\
(0,028)\end{array}$ & 1,098 & 0,003 & $0,110(0,026)$ & 1,116 & $<0,001$ & $0,022(0,042)$ & 1,023 & 0,598 \\
\hline $\begin{array}{l}\text { Sexo* Violência } \\
\text { comunitária }\end{array}$ & - & & - & - & - & - & $0,131(0,052)$ & -4 & 0,022 \\
\hline
\end{tabular}

Nota. ${ }^{1}$ Coeficiente estimado; ${ }^{2}$ Erro-padrão; ${ }^{3}$ Odds Ratio (Razão de chances); ${ }^{4}$ OR não deve ser calculada pelo exp ( $\beta$ ) do termo de interação (Hosmer \& Lemeshow, 2000).

Na Tabela 2 são apresentadas as razões de chances brutas e ajustadas. Destaca-se a diferença que se encontra com a variável idade que passou a ser significativa no modelo multivariado.

Os resultados apresentados no modelo multivariado auxiliam o entendimento dos efeitos do gênero e da exposição à violência comunitária sobre os sintomas de TEPT. Destaca-se que as crianças menores de 10 anos têm duas vezes mais chances de desenvolverem sintomas deste transtorno que as mais velhas e que quanto maior a exposição à violência comunitária, maiores as chances de surgimento de sintomas de TEPT $(12 \%)$.

No estudo da interação entre as variáveis do modelo estudado, constata-se modificações significativas decorrentes da interação entre violência comunitária e sexo feminino $(p=0,022)$. Os efeitos principais de sexo e vio- lência comunitária passam a ser não significativos quando se introduz o termo de interação; enquanto que a variável idade continua a ser significativa $(\mathrm{OR}=2,053 ; p=0,049)$. Observa-se que a faixa etária mais nova apresenta cerca de 2 vezes mais chances de apresentar sintomas de TEPT que a mais velha (Tabela 3 ).

Sobre o termo de interação, ser do sexo feminino aumenta a chance de ter sintomas de TEPT quando a convivência com a violência comunitária é mais elevada (escala com valores de 10 ou mais; Tabela 3 ). A chance de uma menina vir a ter sintomas de TETP com um escore de 10 é de cerca de 2,8 vezes mais do que a de um menino com a mesma pontuação. Quando esse escore é de 20 a chance da menina é ainda maior (aproximadamente 10 vezes a mais) do que a de um menino sob similar exposição à violência.

Tabela 3

Estimação do Efeito do Sexo Feminino sobre o TEPT Controlando por Exposição à Violência da Comunidade (N=399)

\begin{tabular}{ccc}
\hline Escore violência da comunidade & OR & IC95\% \\
\hline 0 & 0,752 & $0,220-2,567$ \\
5 & 1,450 & $0,606-3,468$ \\
10 & 2,796 & $1,333-5,863 *$ \\
15 & 5,392 & $2,120-13,710^{*}$ \\
20 & 10,398 & $2,792-38,751^{*}$ \\
\hline
\end{tabular}

Nota. ${ }^{*}$ coeficientes estatisticamente significativos. 
A Figura 2 ilustra a presença de violência comunitária segundo sexo e idade das crianças e adolescentes. Percebe-se que o efeito do sexo feminino sobre a chance de ter sintomas de TEPT aumenta substancialmente com o escore da escala de violência comunitária, tanto para os mais velhos quanto para os mais novos. Para meninos o aumento é mais discreto.

As associações entre violência comunitária e TEPT são, portanto, mais fortes no sexo feminino que no masculino em ambos os grupos etários. Porém, este efeito é maior na faixa etária mais nova.
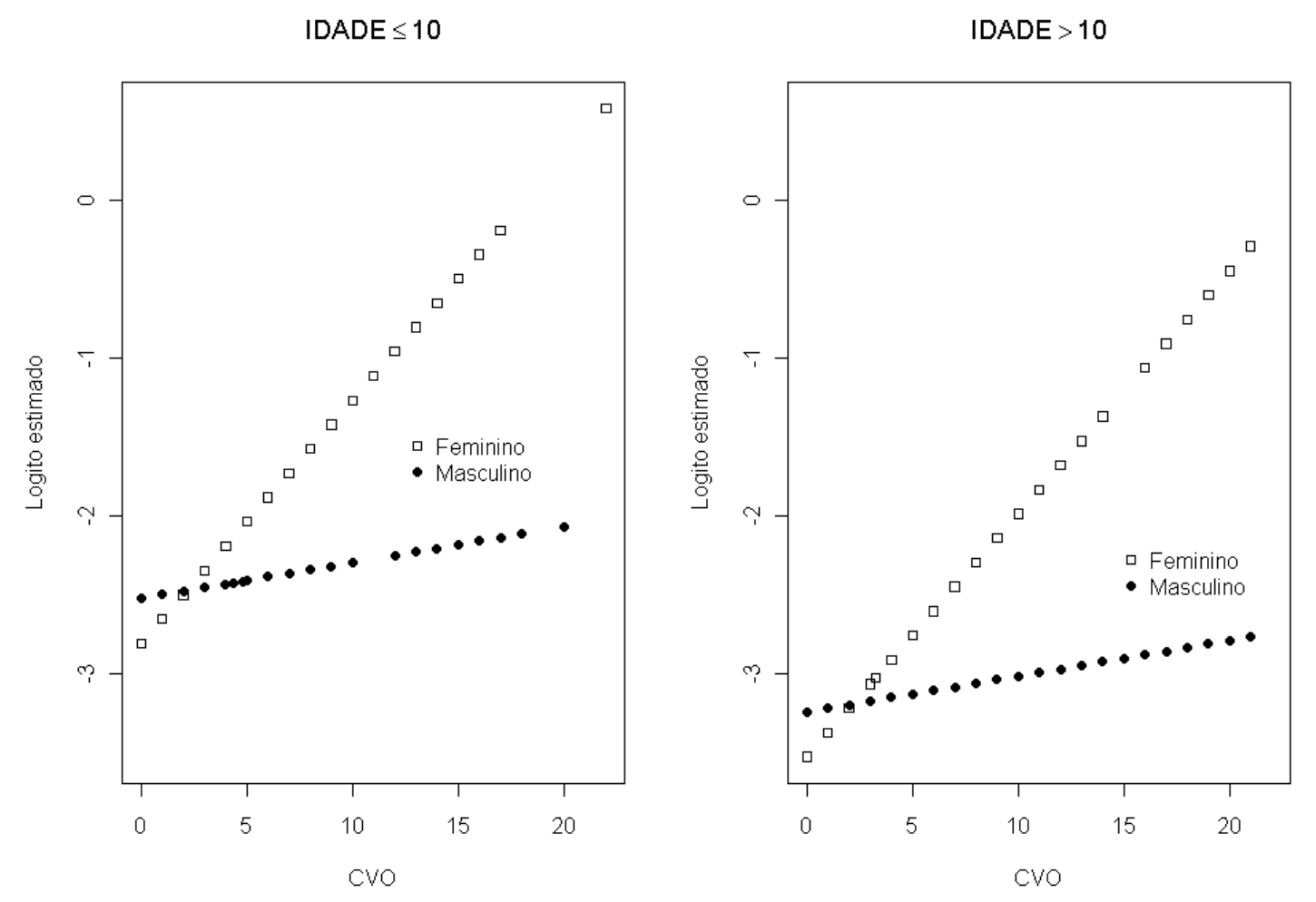

Figura 2. Interação de Violência Comunitária (CVO) e Sexo nos Sintomas de TEPT para crianças e adolescentes com idade menor ou Igual a 10 anos e maiores de 10 anos.

\section{Discussão}

Os resultados mostram que crianças e adolescentes de São Gonçalo estão expostos a elevados níveis de violência comunitária (cerca de $86 \%$ dos entrevistados vivenciaram pelo menos um episódio de violência em sua localidade). Em estudo de revisão sobre o tema, Stein, Jaycox, Kataoka, Rhodes e Vestal (2003) apresentam percentuais similares na população infanto-juvenil dos Estados Unidos: $82 \%$ reportam a vitimização de violência física na comunidade e $99 \%$ o testemunho desta violência.

Já em relação à indistinção verificada da exposição da violência comunitária entre os sexos, resultados díspares são observados na literatura. Alguns estudos apontam que não há diferenças na exposição à violência comunitária entre meninos e meninas (Aisenberg, Ayon, \& Orozco-Figueroa, 2008; Foster et al., 2004), enquanto outros observam exposição maior em crianças e adolescentes do sexo masculino (Richters \& Martinez, 1993).

A prevalência de $9,5 \%$ de crianças e adolescentes com sintomas de TEPT é ligeiramente mais elevada que a en- contrada em etapa anterior do estudo longitudinal ( $6,5 \%$ no ano de 2005). Contudo, cabe ressaltar que na primeira etapa da pesquisa os informantes foram as mães/responsáveis, enquanto que aqui a fonte da informação são as próprias crianças/adolescentes. Além disso, a aferição dos sintomas três anos depois é fator que modifica a ocorrência do problema, pois novos episódios de eventos traumáticos como a violência podem ter ocorrido neste período, levando a manifestação deste transtorno, bem como agravamento dos sintomas. Tais dados merecem aprofundamento através de análises longitudinais e comparativas entre informantes.

Outrossim, tal como constatado por Foster et al. (2004), encontra-se a associação entre violência comunitária e sintomas do Transtorno de Estresse Pós-Traumático em crianças/adolescentes, apresentando maiores taxas do transtorno entre as meninas. Abanilla (2004), em revisão, também mostra que, em níveis maiores de exposição a eventos traumáticos, meninas apresentam maior risco de desenvolver TEPT do que meninos expostos aos mesmos níveis de trauma; em baixos níveis de exposição ambos os sexos apresentam as mesmas taxas do transtorno. 
Ximenes, L. F., Assis, S. G., Pires, T. O. \& Avanci, J. Q. (2013). Violência Comunitária e Transtorno de Estresse Pós-Traumático em Crianças e Adolescentes.

No estudo de Foster et al. (2004), a associação entre sintomas psicológicos foram similares tanto em quem testemunhou quanto em quem foi vítima da violência comunitária. No entanto, nos meninos as associações foram mais fortes quando eles foram vítimas da violência.

Neste estudo a diferença entre os sexos pode ser explicada pelo fato das meninas tenderem a vivenciar tipos específicos de eventos traumáticos, que podem ser mais patogênicos, como o abuso sexual (Tolin \& Foa, 2006). Porém, os eventos traumáticos vivenciados por meninos e meninas explicam apenas em parte esta diferença. Distinções metodológicas entre os estudos exercem papel de peso na constatação desta diferença, como, por exemplo, o uso de amostras de conveniência com maior presença de mulheres. Especula-se ainda se as diferenças poderiam estar ligadas as expectativas sociais relacionadas ao ser homem e ser mulher. Os homens (adultos, adolescentes e crianças) estão mais ligados à imagem de agressividade, o que pode fazê-los a considerar mais aceitável o evento traumático. Já as mulheres, independente de sua faixa etária, por estarem culturalmente associadas à imagem de passividade e emoção, podem incorporar mais sintomas de ansiedade e/ou depressão de forma socialmente mais aceitável (Butler, 2009; Goodyer, 2001).

A constatação de maior prevalência de TEPT entre as crianças mais novas pode ser explicada pela sua maior dificuldade em expressar seus pensamentos e sentimentos sobre a violência comunitária e outros eventos traumáticos, por serem menos capazes de desenvolver estratégias cognitivas para lidarem com situações difíceis e apresentarem menor habilidade em buscar suporte com adultos (Fowler et al., 2009). Também discute-se que os pais/responsáveis podem subestimar a exposição dos filhos mais novos a exposição à violência comunitária e por isso não agem de forma a auxiliá-los a lidar com o que sentem frente a estas situações. Outra explicação é que crianças mais velhas ou adolescentes, por terem vivenciado mais violência comunitária, podem ter se dessensibilizado ao longo do tempo ou ter desenvolvido maiores habilidades para lidar com o problema.

Percebe-se uma vulnerabilidade maior entre as crianças menores que dez anos na apresentação de sintomas do Transtorno de Estresse Pós-Traumático e nas crianças do sexo feminino. A prevalência destes sintomas foi maior entre as meninas, concordando com os dados encontrados na literatura internacional sobre este problema de saúde mental. A associação entre violência comunitária e o TEPT foi mais forte para o sexo feminino tanto no grupo de crianças menores ou maiores que dez anos.

Estes achados permitem pensar que nas situações onde crianças e adolescentes são vítimas de violência comunitária, os profissionais de saúde, educação e outros que lidam com este público devem ficar atentos à possibilidade de desenvolvimento de sintomas do Transtorno de Estresse Pós-Traumático.

Ao notar que crianças e adolescentes estejam desenvolvendo sintomas característicos deste transtorno, é importante o encaminhamento ao tratamento de saúde mental para avaliação e tratamento. A deteç̧ão precoce de sintomas de TEPT e consequentemente o tratamento em fase inicial pode proporcionar um suporte adequado a crianças e suas famílias. Isso pode repercutir numa melhora nos sintomas e no funcionamento geral da criança e do adolescente, bem como impedir o agravamento do problema.

Como principal limitação deste estudo está o fato de não se utilizar uma escala própria para aferir TEPT. Assim, os resultados devem ser tomados como indicativos da presença de sintomas de TEPT em crianças e adolescentes no cenário nacional, porém a adaptação de escalas de screening para o português a serem aplicadas em estudos populacionais sobre TEPT é aspecto relevante para o avanço da pesquisa sobre o tema no país.

Ademais, em futuros estudos, torna-se necessário especificar o grau de exposição à violência comunitária e a quantidade de tempo que a criança/adolescente está exposta a este tipo de violência. É também importante avaliar se a criança/adolescente é vítima, testemunha ou apenas teve conhecimento do evento traumático. O grau de exposição ao evento traumático e a cronicidade dos eventos são indicativos importantes a serem aprofundados em pesquisas na área. É necessária a realização de estudos qualitativos para uma melhor avaliação dos impactos da violência comunitária sobre as crianças e adolescentes, desvelando os sentidos que eles dão a violência vivida ou presenciada.

\section{Referências}

Abanilla, P. K. (2004). Gender differences in childhood PTSD. In R. R. Silva (Ed.), Posttraumatic stress disorders in children $\&$ adolescents: Handbook (pp. 163-176). New York: Norton \& Company.

Achenbach, T. M., \& Rescorla, L. A. (2001). Manual for the ASEBA School-Age Forms e Profiles. Burlington, VT: Research Center for Children, Youth, \& Families, University of Vermont.

Aisenberg, E., Ayon, C., \& Orozco-Figueroa, A. (2008). The role of young adolescents' perception in understanding the severity of exposure to community violence and PTSD. Journal of Interpersonal Violence, 23(11), 1555-1578.

Assis, S. (2009). Violência urbana e problemas de saúde mental infantil. Proposta de análise epidemiológica e espacial em escolares de São Gonçalo/RJ (Relatório de pesquisa). Rio de Janeiro, RJ: Fundação Oswaldo Cruz.

Assis, S. G., Avanci, J. Q., Pesce, R. P., Oliveira, R. V. C., \& Ximenes, L. F. (2007). A violência familiar produzindo reversos: Problemas de comportamento em crianças escolares (Relatório de pesquisa). Rio de Janeiro, RJ: Fundação Oswaldo Cruz.

Assis, S. G., Avanci, J. Q., \& Oliveira, R. V. C. (2009). Desigualdades socioeconômicas e saúde mental infantil. Revista de Saúde Pública, 43(Suppl. 1), 92-100.

Associação de Psiquiatria Americana. (2002). DSM-IV-TR - Manual Diagnóstico e Estatístico de Transtornos Mentais (4. ed. rev.). Porto Alegre, RS: Artmed. 
Bordin, I. A. S., Mari, J. J., \& Caeiro, M. F. (1995). Validação da versão brasileira do "Child Behavior Checklist (CBCL)" (Inventário de comportamentos da Infância e Adolescência): Dados preliminares. Revista ABP-APAL, 17(2), 55-66.

Borges, J. L., \& Dell'Aglio, D. D. (2008). Relações entre abuso sexual na infância, transtorno de estresse pós-traumático (TEPT) e prejuízos cognitivos. Psicologia em Estudo, 13(2), 371-379.

Brasil, H. H. A. (2003). Desenvolvimento da versão brasileira da KSADS- PL (Schedule for Affective Disorders and Schizophrenia for School aged children present and lifetime version) e estudo de suas propriedades psicométricas (Tese de doutorado, Escola Paulista de Medicina, Universidade Federal de São Paulo, SP, Brasil).

Butler, J. (2009). Problemas de gênero: Feminismo e subversão de identidade. Rio de Janeiro, RJ: Civilização Brasileira.

Cardia, N. (1999). Pesquisa sobre atitudes, normas culturais e valores em relação a violência em 10 capitais brasileiras. Brasília, DF: Ministério da Justiça.

Foster, J. D., Kuperminc, G. P., \& Price, A. W. (2004). Gender differences in posttraumatic stress and related symptoms among inner-city minority youth exposed to community violence. Journal of Youth and Adolescence, 33(1), 59-69.

Fowler, P. J., Tompsett, C. J., Braciszewski, J. M., Jacques-Tiura, A. J., \& Baltes, B. B. (2009). Community violence: A metaanalysis on the effect of exposure and mental health outcomes of children and adolescents. Development and Psychopathology, 21(1), 227-259.

Gabbay, V., Oatis, M. D., Silva, R. R., \& Hirsch, G. S. (2004). Epidemiological aspects of PTSD in children and adolescents. In R. R. Silva (Ed.), Posttraumatic stress disorders in children \& adolescents: Handbook (pp. 1-17). New York: Norton \& Company.

Goodyer, I. (2001). The depressed child and adolescent. Cambridge, UK: Cambridge University Press.

Hosmer, D. W., \& Lemeshow, S. (2000). Applied Logistic Regression. New York: John Wiley \& Sons.

Malik, N. M. (2008). Exposure to domestic and community violence in a nonrisk sample: Associations with child functioning. Journal of Interpersonal Violence, 23(4), 490-504.

March, J., Amaya-Jackson, L., Terry, R., \& Constanzo, P. (1997). Postraumatic symptomatology in children and adolescents after an industrial fire. Journal of the American Academy of Child \& Adolescence Psychiatry, 36(8), 1080-1088.

Margolin, G., \& Gordis, E. B. (2000). The effects of family and community violence on children. Annual Review of Psycho$\log y, 51,445-479$.

Passarela, C. M., Mendes, D. D., \& Mari, J. J. (2010). Revisão sistemática para estudar a eficácia de terapia cognitivo-comportamental para crianças e adolescentes abusadas sexualmente com transtorno de estresse pós-traumático. Revista de Psiquiatria Clínica, 37(2), 60-65.

Pessoa, D. G. C., Nascimento Silva, P. L., \& Duarte, R. P. N. (1997). Análise estatística de dados de pesquisas por amostragem: Problemas no uso de pacotes padrões. Revista Brasileira de Estatística, 58(210), 53-75.

Richters, J. E., \& Martinez, P. (1990). Things $i$ have seen and heard: A structured interview for assessing young children's violence exposure. Washington, DC: National Institute of Mental Health.

Richters, J. E., \& Martinez, P. (1993). The NIMH community violence project: I. Children as victims of and witnesses to violence. Psychiatry, 56(1), 7-21.
Ruggiero, K. J., \& McLeer, S. V. (2000). PTSD scale of the Child Behavior Checklist: Concurrent and discriminant validity with non-clinic-referred sexually abused children. Journal of Traumatic Stress, 13(2), 287-299.

Salzinger, S., Feldman R. S., Stockhammer, T., \& Hood, J. (2002). An ecological framework for understanding risk for exposure to community violence and the effects of exposure on children and adolescents. Aggression and Violent Behavior, 7, 423-451.

Scheeringa, M. S. (2008). Developmental considerations for diagnosing PTSD and acute stress disorder in preschool and school-age children. American Journal of Psychiatry, 165(10), 1237-1239.

Scheeringa, M. S., Zeanah, C. H., \& Cohen, J. A. (2010). PTSD in children and adolescents: Toward an empirically based algorithm. Depress and Anxiety, 1-13.

Shields, N., Nadasen, K., \& Pierce, L. (2008). The effects of community violence on children in Cape Town, South Africa. Child Abuse \& Neglect, 32(5), 589-601.

Sieger, K., Rojas-Vilches, A., Mckinney, C., \& Renk, K. (2004). The effects and treatment of community violence in children and adolescents: What should be done? Trauma, Violence \& Abuse, 5(3), 243-259.

Stein, B. D., Jaycox, L. H., Kataoka, S., Rhodes, H. J., \& Vestal, K. D. (2003). Prevalence of child and adolescent exposure to community violence. Clinical Child and Family Psychology Review, 6(4), 247-264.

Tolin, D. F., \& Foa, E. (2006). Sex differences in trauma and posttraumatic stress disorder: A quantitative review of 25 years of research. Psychological Bulletin, 132(6), 959-992.

Wechsler, D. (2002). WISC-III: Escala de inteligência Wechsler para crianças: Manual-Adaptação e padronização brasileira. São Paulo, SP: Casa do Psicólogo.

Wolfe, V. V., Gentile, C., \& Wolfe, D. A. (1989). The impact of sexual abuse on children: A PTSD formulation. Behavior Therapy, 20, 215-228.

Ximenes, L. F., Assis, S. G., \& Oliveira, R. V. C. (2009). Violência e transtorno de estresse pós-traumático na infância. Ciência \& Saúde Coletiva, 14, 417-434. 